\title{
In Vivo Evidence of Enhancement of HGF-induced Angiogenesis by Fluvastatin
}

\author{
Yasushi Takeya $^{1,2}$, Hirofumi Makino ${ }^{1,3}$, Motokuni Aoki ${ }^{4}$, Takashi Miyake ${ }^{1}$, Kazunari Ozaki ${ }^{1,2}$, \\ Hiromi Rakugi ${ }^{2}$, Toshio Ogihara ${ }^{2}$ and Ryuichi Morishita*,1
}

${ }^{I}$ Department of Clinical Gene Therapy, and ${ }^{2}$ Department of Geriatric Medicine and Nephrology, Graduate School of
Medicine, Osaka University, Suita, Japan, ${ }^{3}$ Department of General Medicine, Osaka University Hospital, Suita, Japan
and ${ }^{4}$ Department of Physical Therapy, Morinomiya University of Medical Sciences, Osaka, Japan

\begin{abstract}
Currently, therapeutic angiogenesis using gene transfer of angiogenic growth factors has entered the realm of novel therapy for patients with critical limb ischemia. However, its clinical utility might be enhanced by combination with classical pharmacological approaches. Thus, in this study, we examined adjunctive therapy to enhance the angiogenic effects of gene transfer, with HMG-CoA reductase inhibitors, statins, which have pleiotropic vascular-protective effects. Here, we evaluated the effects of a combination of hepatocyte growth factor (HGF) gene therapy with fluvastatin in a mouse hindlimb ischemia model.

Hindlimb ischemia model mice were given a normal diet, high-cholesterol diet (HCD) or HCD with fluvastatin, after intramuscular injection of human HGF plasmid DNA. Intramuscular injection of HGF plasmid into ischemic limbs significantly increased blood flow and capillary density. However, HCD diminished the increase in blood flow and capillary density induced by HGF gene transfer. Administration of fluvastatin significantly attenuated the reduction in blood flow and decrease in capillary density, while it did not change the serum cholesterol level.

Overall, these results demonstrated that fluvastatin significantly enhanced the increase in blood flow and capillary density induced by HGF gene transfer in a hindlimb ischemia mouse model with HCD, through a non-cholesterol-lowering effect. Clinical use of fluvastatin might be possible adjunctive therapy to enhance therapeutic angiogenesis.
\end{abstract}

Key Words: Angiogenesis, gene therapy, HGF, statin, critical limb ischemia.

\section{INTRODUCTION}

The clinical consequences of peripheral arterial disease (PAD) include pain on walking, claudication, pain at rest and loss of tissue integrity in the distal ischemic limbs. Despite the development of technology for vascular intervention and drugs such as prostaglandins, more than a few patients are still resistant to conventional therapy, and worsening of disease and limb amputation are unavoidable for them. In these circumstances, therapeutic angiogenesis using genes of angiogenic growth factors is expected to be a new treatment for patients with severe ischemic disease. Recent progress in molecular biology has led to the development of gene therapy as a new strategy to treat a variety of cardiovascular diseases using angiogenic growth factors such as vascular endothelial growth factor (VEGF) [1], basic fibroblast growth factor (FGF) [2], and hepatocyte growth factor (HGF) [3, 4]. Several clinical trials of gene therapy using these angiogenic factors have been performed [5-10]. Although some clinical trials using the VEGF121 gene [11] or FGF4 gene [12] did not show a significant improvement in clinical endpoints, we conducted a phase I/IIa clinical trial of gene therapy using HGF [10], which showed that direct intramuscular gene

\footnotetext{
*Address correspondence to this author at the Department of Clinical Gene Therapy, Graduate School of Medicine, Osaka University, 2-2 Yamada-oka, Suita 565-0871, Japan; Tel: +81-6-6879-3406; Fax: +81-6-6879-3409;

E-mail: morishit@cgt.med.osaka-u.ac.jp
}

transfer of naked plasmid DNA encoding human HGF in patients with critical limb ischemia was safe, increased ankle-branchial pressure index, and improved clinical symptoms such as ischemic ulcers. Moreover, a placebo doubleblinded multi-center phase III clinical trial demonstrated significant improvement of the primary endpoints (resting pain and ischemic ulcer). An HGF gene therapy drug is now under new-drug application in Japan, and will be launched in 2009-2010. Nevertheless, it can be anticipated that therapeutic angiogenesis might be enhanced by combination with classical pharmaceutical drugs to treat patients with critical limb ischemia. Thus, it is important to evaluate supplementary and practical treatment in gene-transfer-based angiogenesis.

In this study, we focused on 3-hydroxy-3-methylglutarylcoenzyme A (HMG-CoA) reductase inhibitors, statins, which are used as lipid-lowering agents. Statins are reported to have lipid-independent beneficial effects including improvement of endothelial function [13] and inhibition of inflammation [14]. Moreover, it is recently reported that statins enhance collateral vessel development in the ischemic muscle of animal models $[15,16]$. However, the effects of statins on therapeutic angiogenesis gene therapy have not been elucidated. Here, we investigated the effects of fluvastatin, which is well known as a vascular statin, on peripheral blood flow in a hypercholesterolemic condition and on angiogenesis induced by HGF in a mouse hindlimb ischemia model. 


\section{MATERIALS AND METHODS}

\section{Mouse Ischemic Hindlimb Model}

Ten-week-old male C57/BL6 mice were purchased from Charles River Breeding Laboratories (Yokohama, Japan). All animal experiments were conducted under protocols approved by the Institutional Animal Care Committee of Osaka University. Animals were anesthetized with an intraperitoneal injection of sodium pentobarbital (30mg/ $\mathrm{kg}$ body weight). A longitudinal incision was then made, extending inferiorly from the inguinal ligament to a point just proximal to the patella. Through this incision, using surgical loupes, the operator dissected free the left femoral artery along its entire length; all branches of the femoral artery, including the inferior epigastric, deep femoral, lateral circumflex, and superficial epigastric arteries, were also dissected free. After dissection of the popliteal and saphenous arteries distally, the external iliac artery and all of the mentioned arteries were ligated with 4-0 silk. Finally, the right femoral artery was completely excised to create the ischemic limb model, from its proximal origin as a branch of the external iliac artery to the point distally where it bifurcates to form the saphenous and popliteal arteries. Excision of the femoral artery results in retrograde propagation of thrombus and occlusion of the external iliac artery. Excision of the femoral artery $1 \mathrm{~cm}$ below the peritoneum created an ischemia model. Consequently, blood flow to the ischemic limb was dependent on collateral vessels developing from the internal iliac artery.

\section{Administration of Fluvastatin}

Mice were divided into four groups; (1) control group fed a normal diet (ND), (2) high-cholesterol diet (HCD), (3) $\mathrm{HCD}+1 \mathrm{mg} / \mathrm{kg} /$ day fluvastatin, (4) $\mathrm{HCD}+5 \mathrm{mg} / \mathrm{kg} / \mathrm{day}$ fluvastatin. At 1 week after surgery, high-cholesterol diet (containing $1.25 \%$ cholesterol) and fluvastatin (Novartis Pharma Japan, Tokyo, Japan) were started. Fluvastatin was continuously administered by an osmotic mini-pump at a dose of 1 or $5 \mathrm{mg} / \mathrm{kg} /$ day. After 4 weeks of high-cholesterol diet and fluvastatin, blood flow and capillary density in the hindlimb were measured. Plasma level of total cholesterol was also measured.

\section{In Vivo Gene Transfer to Ischemic Limb Model Using Direct Intramuscular Injection}

To produce an HGF expression vector, human $\mathrm{HGF}$ cDNA (2.2 kbp) was inserted into a simple eukaryotic expression plasmid that utilizes the cytomegalovirus promoter/enhancer [4, 17]. This promoter/enhancer has been used to express reporter genes in a variety of cell types and can be considered to be constitutive. Cytomegalovirus expression vector plasmid without HGF cDNA sequence was used as a control vector.

We injected $500 \mu \mathrm{g}$ naked plasmid DNA directly into the ischemic limb of mice with a $27 \mathrm{G}$ needle (Terumo, Tokyo, Japan) at 1 week after surgery. The injection volume of plasmid was $100 \mu \mathrm{L}$. After gene transfection, high-cholesterol feeding and administration of fluvastatin were performed as described above.

\section{Measurement of Blood Flow by Laser Doppler Imager}

Measurement of blood flow with a laser Doppler imager (LDI) has been previously described $[4,17]$. As it is clearly demonstrated that laser Doppler flow velocity correlates well with capillary density, we measured blood flow using a laser Doppler imager blood flowmeter (Moor Instruments, England). Excess hairs were removed from the limb with depilatory cream before imaging, and rats were placed on a heating plate at $37^{\circ} \mathrm{C}$ to minimize temperature variation. Consecutive measurements were obtained over the same regions of interest (leg and foot). The LDI uses a 12-mW helium-neon laser beam that sequentially scans a $5 \times 5 \mathrm{~cm}$ surface area at extremely high speed to be able to measure blood flow in the ischemic hindlimb. Blood flow at $1 \mathrm{~mm}$ under the surface can be measured. While scanning, the motion shift of blood cells is converted to the frequency of projected light according to the Doppler principle. Upon the termination of scanning, a color-coded image representing blood flow distribution is displayed on a monitor. The perfusion signal is subdivided into 14 different intervals, and each interval is displayed as a separate color. Low or no perfusion is displayed as dark blue, whereas the highest perfusion interval is displayed as white. The stored perfusion values behind the color-coded pixels remain available for data analysis. These laser images were quantitatively converted into histograms with blood flow on the $\mathrm{x}$-axis and the number of pixels on the $y$-axis in the traced area. The average blood flow in each histogram was calculated for evaluation.

\section{Measurement of Capillary Density}

Alkaline phosphatase staining was used as a specific marker for endothelial cells in paraffin-embedded sections, as previously described $[4,17]$. To analyze the number of vessels in the ischemic hindlimb transfected with HGF vector or control vector, mice were sacrificed and the muscles were removed. Three individual sections from the middle of the transfected muscle were analyzed. The number of vessels was counted under light microscopy (magnification, x100) in a blinded manner. The total number of vessels) in each section was summed and expressed as the number per section. At least ten individual sections were evaluated in each muscle. The areas in which the number of vessels was quantified were randomly selected in the injected site and around the injected site. The animals were coded so that analysis was performed without any knowledge of which treatment each animal had received. The reproducibility of the results was assessed.

Table 1. Cholesterol Levels in Mice Treated with Vehicle or Fluvastatin

\begin{tabular}{|c|c|}
\hline & Total Cholesterol (mg/dL) \\
\hline \hline 1) ND & $83 \pm 10$ \\
\hline 2) HCD & $177 \pm 23 \#$ \\
\hline 3) HCD+Flu 1 & $180 \pm 18 \#$ \\
\hline 4) HCD+Flu 5 & $164 \pm 16 \#$ \\
\hline
\end{tabular}

\# p $<0.05$ vs ND, ND = ischemic hindlimb mice fed normal diet, HCD = ischemic hindlimb mice fed high-cholesterol diet, HCD+Flu $1=$ ischemic hindlimb mice fed high-cholesterol diet with fluvastatin $(1 \mathrm{mg} / \mathrm{kg} /$ day $), \mathrm{HCD}+$ Flu $5=$ ischemic hindlimb mice fed high-cholesterol diet with fluvastatin ( $5 \mathrm{mg} / \mathrm{kg} / \mathrm{day})$. Each group contains 5-7 animals. 


\section{Statistical Analysis}

All values are expressed as mean + SEM. Analysis of variance with subsequent Duncan's test was used to determine the significance of differences in multiple comparisons. Differences with a $\mathrm{p}$ value less than 0.05 were considered significant.

\section{RESULTS}

\section{Effect of Fluvastatin on Serum Cholesterol Level}

Initially, we measured plasma cholesterol level after administration of fluvastatin at 1 and $5 \mathrm{mg} / \mathrm{kg} /$ day to mice fed a high-cholesterol diet. As shown in Table 1, these doses of fluvastatin did not affect serum total cholesterol level.

\section{Effects of Fluvastatin on Peripheral Blood Flow}

To assess the effects of a high-cholesterol diet and fluvastatin on recovery of peripheral blood flow, we measured peripheral perfusion in the ischemic limb using a laser Doppler imager. As shown in Fig. (1a) \& (1b), peripheral perfusion ratio was significantly reduced in mice fed HCD as compared to those with a normal diet. Interestingly, administration of fluvastatin alone attenuated the reduction of blood flow by HCD in a dose-dependent manner (Fig. 1b), although a low dose of fluvastatin did not affect peripheral blood flow. Perfusion ratio was significantly increased in mice administered a high dose of fluvastatin as compared to those with HCD only.

\section{Enhancement of HGF-Induced Blood Flow Recovery and Angiogenesis by Fluvastatin}

Next, we performed transfection of the human HGF gene to the ischemic limb. In the normal diet group, overexpression of the human HGF gene resulted in a significant increase in blood flow in the ischemic hindlimb as compared to control vector (Fig. $\mathbf{2 a}$ and $\mathbf{2 b}$ ), consistent with our previous data $[4,17]$. Interestingly, the recovery of blood flow was significantly diminished in hypercholesterolemic mice as compared to those with a normal diet (Fig. 2-b). Importantly, administration of fluvastatin enhanced the ?increase in blood flow induced by HGF in a dose-dependent manner.

Angiogenesis was also confirmed by measurement of capillary density as assessed by alkaline phosphatase staining. As shown in Fig. (3), capillary density after 4 weeks of HCD was also significantly lower in hypercholesterolemic mice than in mice fed a normal diet, although transfection of human HGF plasmid DNA significantly increased the capillary density. Importantly, capillary density was significantly higher in mice treated with a high dose of fluvastatin as compared to mice without fluvastatin (Fig. 3). These data suggest that fluvastatin enhanced HGF-induced angiogenesis in a hypercholesterolemic condition. $\mathbf{a}$

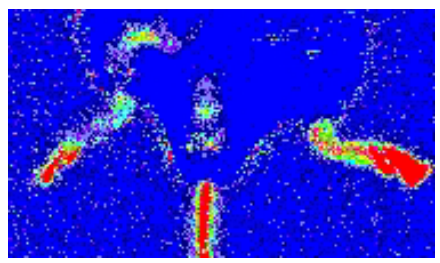

Control

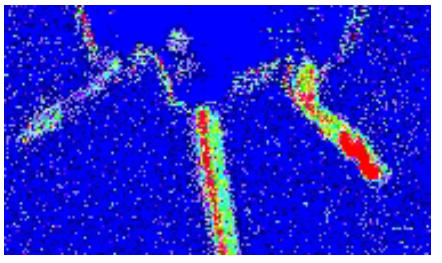

HCD

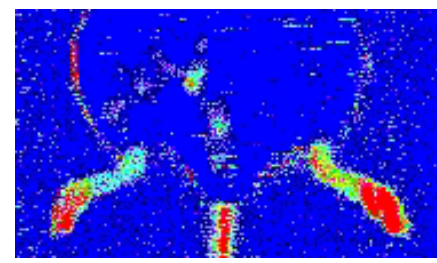

HCD

b

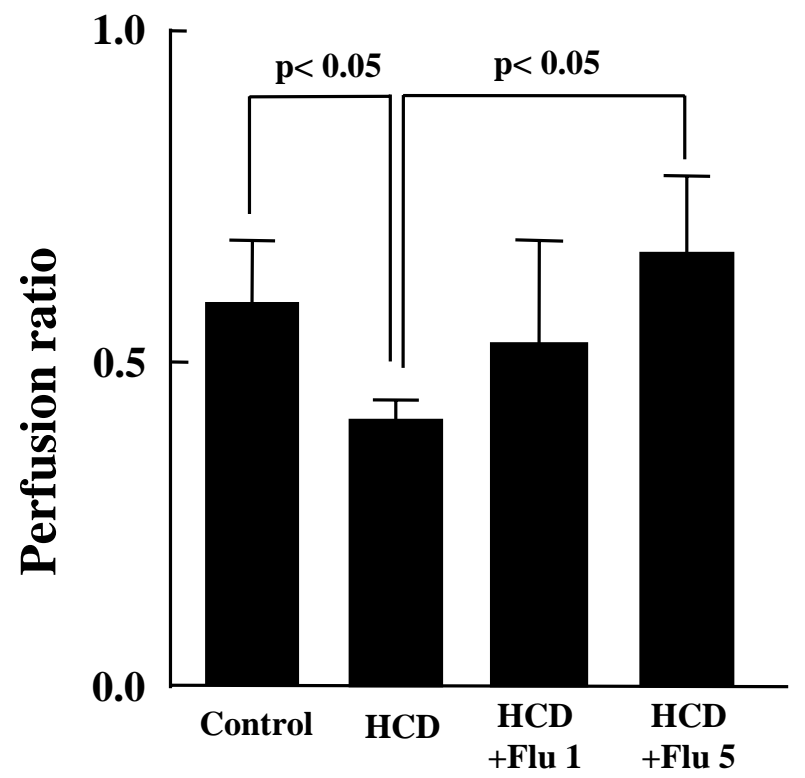

Fig. (1). Effect of 4 weeks of high-cholesterol diet and/or fluvastatin on recovery of blood flow in mouse ischemic hindlimb model. (1-a). Representative photographs of LDI. (1-b). Quantitative analysis of blood flow in hindlimb expressed as perfusion ratio of ischemic hindlimb to untreated opposite limb. ND = normal diet, $\mathrm{HCD}=$ high-cholesterol diet, HCD+Flu 1 = high-cholesterol diet with fluvastatin (1 $\mathrm{mg} / \mathrm{kg} /$ day $)$, HCD+Flu 5 = high-cholesterol diet with fluvastatin $(5 \mathrm{mg} / \mathrm{kg} / \mathrm{day})$. Each group contains $5-7$ animals. 
$\mathbf{a}$

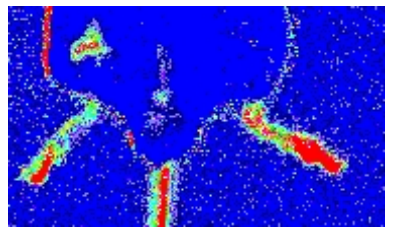

ND

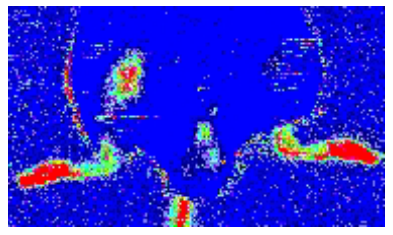

ND+HGF

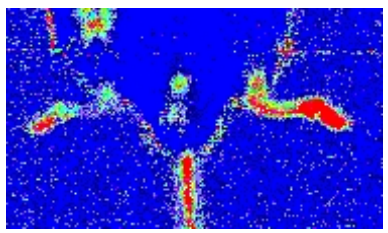

HCD+HGF

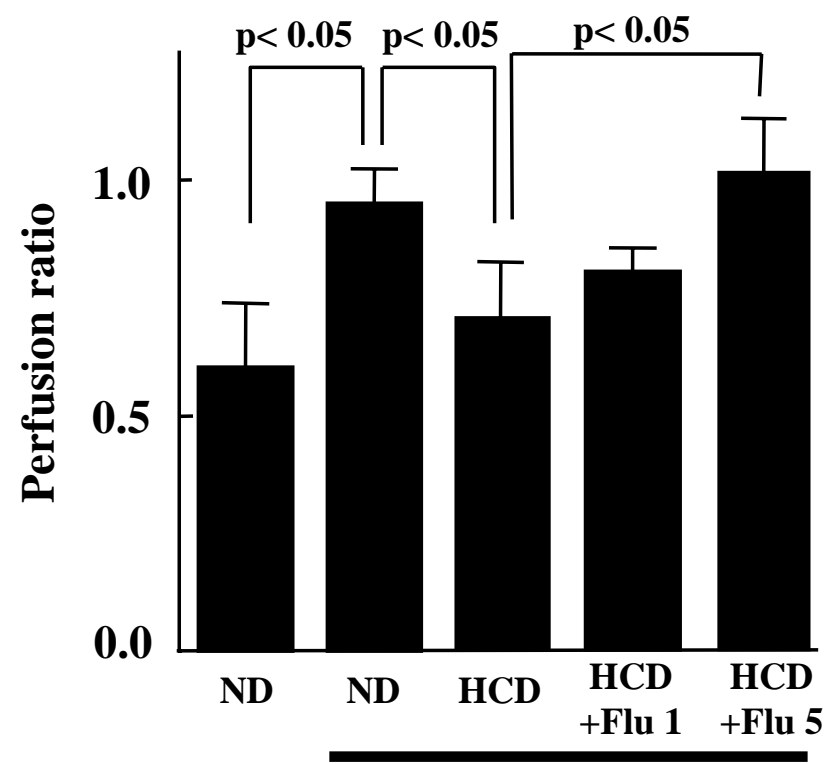

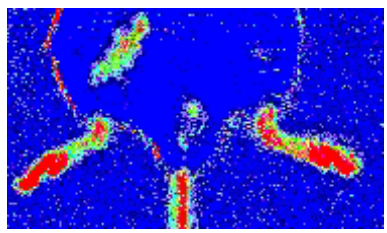

HCD+HGF +Flu 5

b

\section{HGF}

Fig. (2). Effect of fluvastatin on increase in blood flow induced by transfection of human HGF gene in ischemic hindlimb mouse model at 4 weeks after transfection. (2-a) Representative photographs of LDI. (2-b) Quantitative analysis of blood flow in hindlimb expressed as perfusion ratio of ischemic hindlimb to untreated opposite limb. ND $=$ normal diet, $\mathrm{HCD}=$ high-cholesterol diet, HCD + Flu $1=$ high-cholesterol diet with fluvastatin $(1 \mathrm{mg} / \mathrm{kg} / \mathrm{day})$, HCD+Flu 5 = high-cholesterol diet with fluvastatin $(5 \mathrm{mg} / \mathrm{kg} / \mathrm{day})$, HGF = human HGF plasmid (500 $\mu \mathrm{g})$. Each group contains 5-7 animals.

\section{DISCUSSION}

Critical limb ischemia is estimated to develop in 220 persons per million per year [18], and the natural history of critical limb ischemia has been well documented to be an inexorable downhill course $[19,20]$. Therapeutic angiogenesis using angiogenic growth factors should be considered for the treatment of patients with critical limb ischemia. Several growth factors such as HGF, VEGF, FGF and others have been tested in clinical trials. The first gene therapy drug to stimulate angiogenesis will be launched within a few years, since HGF gene therapy trials have been successfully completed and a FGF phase III trial is now ongoing. However, angiogenesis induced by single gene transfer of an angiogenic growth factor might be not ideal for patients who have multiple risk factors for atherosclerosis and severe critical limb ischemia. Thus, to consider the practical and clinical situation, additional treatment to enhance angiogenesis should be investigated.

In the present study, we employed fluvastatin as an adjunct treatment combined with therapeutic angiogenesis induced by HGF, because statins are well known to improve endothelial function independently of their lipid-lowering effect [13]. Hypercholesterolemia induces endothelial dys- function through vascular inflammation and/or oxidative stress [21, 22], and leads to a delay of angiogenesis in ischemic lesions. Indeed, endothelial dysfunction in hypercholesterolemic animals results in poor collateral formation and a delay of recovery of peripheral blood flow in ischemic lesions [23, 24]. Endothelial function is closely related to collateral formation. Given that more than $50 \%$ of patients with peripheral arterial disease have hypercholesterolemia [25], restoring endothelial dysfunction is an important factor in considering treatment for atherosclerotic disease. Thus, statins are expected to be suitable for concomitant use with therapeutic angiogenesis. Especially, fluvastatin is well known as a vascular statin, and might have superior effects by a direct action on the blood vessel wall.

The present study demonstrated that administration of fluvastatin enhanced angiogenesis either alone or in combination with HGF in the ischemic hindlimb under a hypercholesterolemic condition. As fluvastatin did not affect serum cholesterol level, these effects might be independent of its lipid-lowering effect. It is reported that statins promote ischemia-induced angiogenesis through induction of endothelial nitric oxide synthase (eNOS) gene expression [15, 16]. Statins have been shown to promote angiogenesis via 
up-regulation of eNOS expression [26, 27], activation of Akt [28] or inactivate Rho [29]. In addition, transfection of the eNOS gene induces angiogenesis in ischemic muscle through up-regulation of VEGF [30]. Moreover it is also reported that statins up-regulate VEGF expression and augment proliferation of microvessels in diabetic mice model [31].

\section{vessels $/ \mathbf{m m}^{2}$}

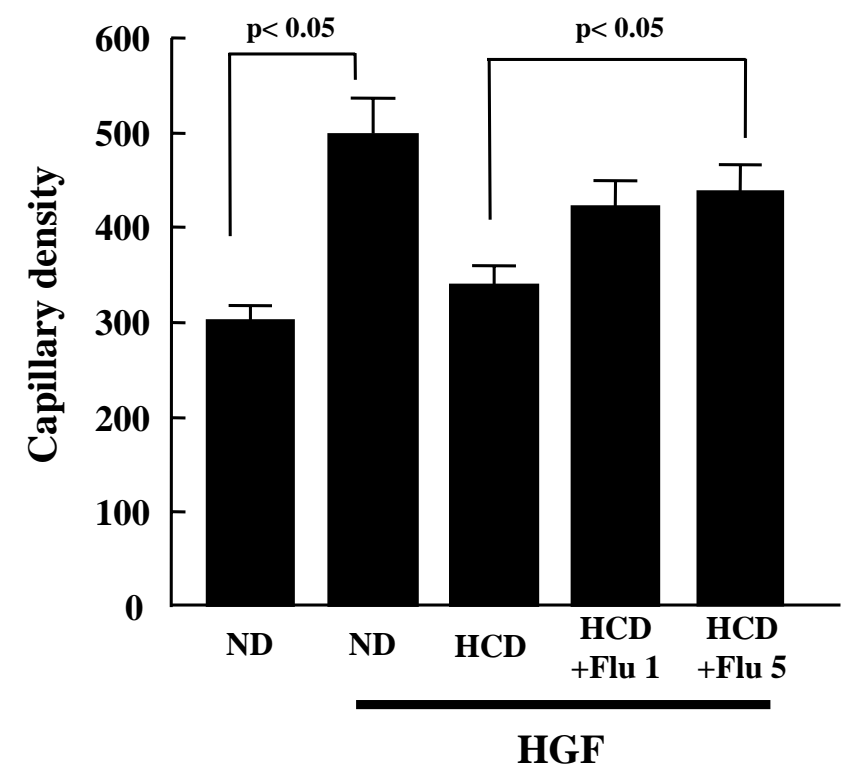

Fig. (3). Effect of transfection of HGF plasmid on capillary density in ischemic hindlimb of mice at 4 weeks after transfection. ND = normal diet, $\mathrm{HCD}=$ high-cholesterol diet, HCD+Flu 1 = highcholesterol diet with fluvastatin $(1 \mathrm{mg} / \mathrm{kg} /$ day $), \mathrm{HCD}+\mathrm{Flu} 5=$ highcholesterol diet with fluvastatin $(5 \mathrm{mg} / \mathrm{kg} / \mathrm{day}), \mathrm{HGF}=$ human $\mathrm{HGF}$ plasmid $(500 \mu \mathrm{g})$. Each group contains 5-7 animals.

Furthermore, statins may improve blood flow through vasodilatation induced by its effect of improvement in endothelial function. Statins improve endothelial dysfunction through various mechanisms without lipid-lowering effects. One is their anti-inflammatory effect. Vascular inflammation is regarded as important in the pathogenesis of atherosclerosis. Statins are reported to inhibit inflammation in the vascular system. This concept is supported by recent findings that statins decreased high-sensitive $\mathrm{C}$ reactive protein $[32,33]$ and inhibited the progression of atherosclerosis [34]. It is also reported that statins attenuated activation of the transcription factor nuclear factor-kappa B (NF-kB), which regulates various cytokines, chemokines and adhesion molecules to cause adhesion of monocytes, neutrophils and macrophages [35]. Moreover, as mentioned above, statins upregulate eNOS gene expression [26, 27], which induce an increase in $\mathrm{NO}$ and may subsequently lead to vasodilatation. Both of the mechanisms, vasodilating effect via an improvement in endothelial function and angiogenic effect, could contribute to augmentation of blood flow.

\section{CONCLUSION}

Here, the present study demonstrated that the combination of fluvastatin with HGF gene transfer enhanced the increase in peripheral blood flow induced by HGF in a hyper- cholesterolemic condition. These data suggest that statins such as fluvastatin might have therapeutic potential for supplementary treatment in therapeutic angiogenesis.

\section{ACKNOWLEDGEMENT}

We thank Kiyomi Yamamoto for her excellent technical assistance. This work was partially supported by grants from the Japan Cardiovascular Research Foundation and a Grantin-Aid from the Ministry of Education, Science, Sports and Culture.

\section{REFERENCES}

[1] Tsurumi Y, Takeshita S, Chen D, et al. Direct intramuscular gene transfer of naked DNA encoding vascular endothelial growth factor augments collateral development and tissue perfusion. Circulation 1996; 94: 3281-90.

[2] Giordano FJ, Ping P, McKirnan MD, et al. Intracoronary gene transfer of fibroblast growth factor-5 increases blood flow and contractile function in an ischemic region of the heart. Nat Med 1996; 2: 534-9.

[3] Morishita R, Nakamura S, Hayashi S, et al. Therapeutic angiogenesis induced by human recombinant hepatocyte growth factor in rabbit hind limb ischemia model as cytokine supplement therapy Hypertension 1999; 33: 1379-84.

[4] Taniyama Y, Morishita R, Aoki M, et al. Therapeutic angiogenesis induced by human hepatocyte growth factor gene in rat and rabbit hindlimb ischemia models: preclinical study for treatment of peripheral arterial disease. Gene Ther 2000; 8: 181-9.

[5] Baumgartner I, Pieczek A, Manor O, et al. Constitutive expression of phVEGF165 after intramuscular gene transfer promotes collateral vessel development in patients with critical limb ischemia. Circulation 1998; 97: 1114-23.

[6] Losordo DW, Vale PR, Symes JF, et al. Gene therapy for myocardial angiogenesis: initial clinical results with direct myocardial injection of phVEGF165 as sole therapy for myocardial ischemia. Circulation 1998; 98: 2800-4.

[7] Vale PR, Losordo DW, Milliken CE, et al. Left ventricular electromechanical mapping to assess efficacy of phVEGF165 gene transfer for therapeutic angiogenesis in chronic myocardial ischemia. Circulation 2000; 102: 965-74.

[8] Rosengart TK, Lee LY, Patel SR, et al. Angiogenesis gene therapy: phase I assessment of direct intramyocardial administration of an adenovirus vector expressing VEGF121 cDNA to individuals with clinically significant severe coronary artery disease. Circulation 1999; 100: 468-74.

[9] Grines CL, Watkins MW, Helmer G, et al. Angiogenic Gene Therapy (AGENT) trial in patients with stable angina pectoris. Circulation 2002;105:1291-7.

[10] Morishita R, Aoki M, Hashiya N, et al. Safety evaluation of clinical gene therapy using Hepatocyte Growth Factor to treat peripheral arterial disease. Hypertension 2004; 44: $203-9$.

[11] Rajagopalan S, Mohler ER 3rd, Lederman RJ, et al. Regional angiogenesis with vascular endothelial growth factor in peripheral arterial disease; A phase II randomized, double-blind, controlled study of adenoviral delivery of vascular endothelial growth factor 121 in patients with disabling intermittent claudication. Circulation 2003; 108: 1933-8.

[12] Grines CL, Watkins MW, Mahmarian JJ, et al. A randomized, double-blind, placebo-controlled trial of Ad5FGF-4 gene therapy and its effect on myocardial perfusion in patients with stable angina. J Am Coll Cardiol 2003; 42: 1339-47.

[13] Davingnon J, Laaksonen R. Low-density lipoprotein-independent effects of statins. Curr Opin Lipidol 1999; 10: 543-59.

[14] Maron DJ, Fazio S, Linton MF. Current perspectives on statins. Circulation 2000; 101: 207-13.

[15] Sata M, Nishimatsu H, Suzuki E, et al. Endothelial nitric oxide synthase is essential for the HMG-CoA reductase inhibitor cerivastatin to promote collateral growth in response to ischemia. FASEB J 2001; 15: 2530-2.

[16] Sata M, Nishimatsu H, Osuga J, et al. Statins augment collateral growth in response to ischemia but they do not promote cancer and atherosclerosis. Hypertension 2004; 43: 1214-20.

[17] Morishita R, Sakaki M, Yamamoto K, et al. Inmpairment of collateral formation in lipoprotein(a) transgenic mice: Therapeutic angi- 
ogenesis induced by human hepatocyte growth factor gene. Circulation 2002; 105: 1491-6.

[18] Rothwell PM, Eliasziw M, Gutnikov SA, Warlow CP, Barnett HJ; Carotid Endarterectomy Trialists Collaboration. Endarterectomy for symptomatic carotid stenosis in relation to clinical subgroups and timing of surgery. Lancet 2004; 363: 915-24.

[19] Dormandy J, Mahir M, Ascady G, et al. Fate of the patient with chronic leg ischaemia. A review article. J Cardiovasc Surg (Torino) 1989; 30: 50-7.

[20] Rutherford RB, Flanigan DP, Gupta SK, et al. Suggested standards for reports dealing with lower extremity ischemia. Ad Hoc Committee on Reporting Standards. Society for Vascular Surgery/North Am Chapter, International Society for Cardiovascular Surgery. J Vasc Surg 1986; 4: 80-94.

[21] Creager MA, Cooke JP, Mendelsohn ME, et al. Impaired vasodilation of forearm resistance vessels in hypercholesterolemic humans. J Clin Invest 1990; 86: 226-34.

[22] Chowienczyk PJ, Watts GF, Crockroft JR, Ritter JM. Impaired endothelium-dependent vasodilation of forearm resistance vessels in hypercholesterolemia. Lancet 1992; 340: 1430-2.

[23] Couffinhal T, Silver M, Kearney M, et al. Impaired collateral vessel development associated with reduced expression of vascular endothelial growth factor in ApoE-/- mice. Circulation 1999; 99: 3188-98.

[24] Van Belle E, Rivard A, Chen D, et al. Hypercholesterolemia attenuates angiogenesis but does not preclude augmentation by angiogenic cytokines. Circulation 1997; 96: 2667-74.

[25] Perlstein TS, Pande RL, Beckman JA, Creager MA. Serum total bilirubin level and prevalent lower-extremity peripheral arterial disease: National Health and Nutrition Survey (NANTES) 19992004. Arterioscler Thromb Vasc Biol 2008; 28: 166-72.

[26] Laufs U, La Fata V, Plutzky J, Liao JK. Upregulation of endothelial nitric oxide synthase by HMG CoA reductase inhibitors. Circulation 1998; 97: 1129-35.
[27] Hernadez-Perara O, Perez-Sala D, Navarro-Antolin J, et al. Effects of the 3-hydroxy-3-methylglutalryl-CoA reductase inhibitors, atorvastatin and simvastatin, on the expression of endothelin-1 and endothelial nitric oxide synthase in vascular endothelial cells. J Clin Invest 1998; 101: 2711-9.

[28] Kureishi Y, Luo Z, Shiojima I, et al. The HMG-CoA reductase inhibitor simvastatin activates the protein kinase Akt and promotes angiogenesis in normocholesterolemic animals. Nat Med 2000; 6: 1004-10.

[29] Laufs U, Liao JK. Post-transcriptional regulation of endothelial nitric oxide synthase mRNA stability by Rho GTPase. J Biol Chem 1998; 273: 24266-71.

[30] Namba T, Koike H, Murakami K, et al. Angiogenesis induced by endothelial nitric oxide synthase gene through vascular endothelial growth factor expression in a rat hindlimb ischemia model. Circulation 2003; 108: 2250-7.

[31] Bitto A, Minutoli L, Altavilla D, et al. Simvastatin enhances VEGF production and ameliorates impaired wound healing in experimental diabetes. Pharmacol Res 2008; 57: 159-69.

[32] Ridker PM, Rifai N, Pfeffer MA, Sacks F, Braunwald E. Longterm effects of pravastatin on plasma concentration of C-reactive protein. The Cholesterol and Recurrent Events (CARE) Investigators. Circulation 1999; 100: 230-5.

[33] Albert MA, Danielson E, Rifai N, Ridker PM; PRINCE Investigators. Effect of statin therapy on C-reactive protein levels: the pravastatin inflammation/CRP evaluation (PRINCE): a randomized trial and cohort study. JAMA 2001; 286: 64-70.

[34] Nissen SE, Tuzcu EM, Schoenhagen P, et al. Statin therapy, LDL cholesterol, C-reactive protein, and coronary artery disease. N Engl J Med 2005; 352: 29-38

[35] Dichtl W, Dulak J, Frick M, et al. HMG-CoA reductase inhibitors regulate inflammatory transcription factors in human endothelial and vascular smooth muscle cells. Arterioscler Thromb Vasc Biol 2003; 23: 58-63.

Received: April 23, 2008

Revised: May 09, 2008

Accepted: May 09, 2008

() Takeya et al.; Licensee Bentham Open.

This is an open access article distributed under the terms of the Creative Commons Attribution License (http://creativecommons.org/licenses/by/2.5/), which permits unrestrictive use, distribution, and reproduction in any medium, provided the original work is properly cited. 